\title{
Clinical effectiveness of combination therapy with dulaglutide, SGLT2 inhibitor and metformin with or without insulin in Indian adults with type 2 diabetes: a real-world retrospective study
}

\section{ABSTRACT}

Background. Antidiabetic agents such as glucagon-like peptide-1 receptor agonists (GLP-1RAs) and sodium glucose cotransporter-2 inhibitors (SGLT2i) are known to improve glycaemic control with favourable impact on cardiovascular (CV) risk factors. Given the potential benefits, this study evaluated the clinical effectiveness of combination therapy including dulaglutide, SGLT2 $i$ and metformin with or without insulin in Indian adults with inadequately controlled T2DM.

Material and methods. This retrospective, real-world, single-centre study included 15 adults (mean age [SD, standard deviation]: 49.47 [9.29] years) with inadequately controlled T2DM. The patients received a combination of dulaglutide, SGLT2 $i$ and metformin with or without insulin. Changes in fasting blood glucose (FBG), postprandial blood glucose (PPBG), glycosylated haemoglobin $\left(\mathrm{HbA}_{1 \mathrm{c}}\right)$, body weight, BMI, vascular age (VA), systolic blood pressure (SBP) and diastolic blood pressure (DBP) were examined for a follow-up duration of 3 months. Self-reported adverse events were also recorded.

Results. At the 3-month follow-up, the combination therapy resulted in significant reduction $(P<0.001)$ in

Address for correspondence:

Dr. Supratik Bhattacharyya

Endocrinology and Diabetes, AMRI Hospital

Salt Lake, Kolkata, West Bengal 700098

e-mail: dr_supratik@yahoo.co.uk

Clinical Diabetology 2020, 9, 4, 233-238

DOI: $10.5603 /$ DK.2020.0026

Received: 16.02 .2020

Accepted: 12.06.2020 glycaemic parameters such as FBG, PPBG and $\mathrm{HbA}_{1 \mathrm{c}}$ with a mean reduction (MR [SD]) of $66.67(40.05) \mathrm{mg} / \mathrm{dL}, 83.33$ (64.11) $\mathrm{mg} / \mathrm{dL}$ and 1.78 (1.08) \% respectively. A significant reduction $(P<0.001)$ in body weight and corresponding BMI was demonstrated with an MR (SD) of 6.40 (3.96) kg and $2.67(1.63) \mathrm{kg} / \mathrm{m}^{2}$ respectively. Similarly, significant reductions $(P<0.05)$ were also recorded for SBP (4.40 [4.61] $\mathrm{mm} \mathrm{Hg}$ ) and DBP (2.80 [3.84] $\mathrm{mm} \mathrm{Hg})$. The therapy was also associated with significant reduction $(P<0.001)$ in VA (MR [SD]: 3.93 [2.46] years). The therapy was well tolerated; however, self-reported gastrointestinal symptoms were reported in 5 patients, which subsided within 2 weeks of therapy initiation.

Conclusion. Combination therapy with dulaglutide, SGLT2 $i$ and metformin with or without insulin resulted in significant improvements in glycaemic parameters, body weight and SBP in Indian adults with inadequately controlled T2DM. Interestingly, there was a significant improvement in vascular age associated with the therapy. (Clin Diabetol 2020; 9; 4: 233-238)

Key words: dulaglutide, GLP-1RAs, India, SGLT-2i, T2DM

\section{Introduction}

Type 2 diabetes mellitus (T2DM) is considered a global epidemic affecting nearly $90 \%$ of population world-wide. Patients with T2DM are usually presented with comorbidities including hypertension, hyperlipidaemia, obesity and are therefore prone to cardiovascular (CV) risk [1]. Management of T2DM is complex given the progressive nature of the disease and associated 
co-morbidities. As the disease progresses, clinicians involved in diabetes care often tackle with the challenge of adjusting multiple medications in the face of renal failure and CV disease. In addition, medications have to be tailored to help patients overcome intolerances and adverse effects [2].

Newer generation glucose-lowering drugs, glucagon-like peptide-1 receptor agonists (GLP-1RA) and sodium glucose cotransporter-2 inhibitors (SGLT2i) have made a remarkable breakthrough into the T2DM treatment armamentarium enabling a patient centred approach. The complimentary mechanisms of action demonstrated by GLP-1RA and SGLT2i with respect to cardio and nephroprotective effects are generating their potential need and are currently attracting significant attention from medical research communities [3]. Cardiovascular outcome trials (CVOT) have demonstrated significant reductions in major adverse cardiovascular events (MACE) and mortality outcomes with GLP-1RA (LEADER, EXCEL, SUSTAIN-6) and SGLT2i (EMPA-REG, CANVAS) [4]. To further support this evidence, REWIND, the first CVOT trial for dulaglutide that included a majority of participants who did not have an established cardiovascular disease, is reported to have demonstrated superiority in the reduction of MACE [5]. Based on evidence from pivotal trials (DURATION-8, AWARD-10) and other recently published real-world observational studies, patients with poorly controlled T2DM treated with GLP-1RA as an add-on to SGLT2i have shown beneficial effects on glycaemic control, body weight and SBP with low hypoglycaemia risk compared with those assigned to placebo [1, 4, $6,7]$. A literature review on the efficacy of combined treatment with GLP-1RA and SGLT2i showed additive effect on lowering the $\mathrm{HbA}_{\mathrm{lc}}$ levels, systolic blood pressure (SBP), diastolic blood pressure (DBP) and body weight in patients with T2DM. The combination therapy may therefore address multiple facets of the disease and may prove to be a potential additive in lowering cardiorenal risk in addition to glycaemic control $[1,8]$.

The aim of this retrospective, real-world, singlecentre study is to evaluate the impact of combination therapy with once-weekly dulaglutide, SGLT2i and metformin with or without insulin on fasting blood glucose (FBG), post prandial blood glucose (PPBG), $\mathrm{HbA}_{1 c^{\prime}}$ body weight, body mass index (BMI), vascular age (VA), SBP and DBP in Indian patients with inadequately controlled T2DM in a real-world setting.

\section{Materials and methods}

\section{Study design and participants}

This retrospective, real-world, single-centre observational study was carried out at the AMRI Hospital, a tertiary care centre in Salt Lake, Kolkata, India between January, 2018 to March, 2018. Eligible patients $(N=15)$ were adults (mean age, $49.47 \pm 9.29$ years) with inadequately controlled T2DM. The mean duration of diabetes was $7.6 \pm 1.24$ years. All participants were receiving antidiabetic medications which included a full dose of gliptin (Sitagliptin [100 mg] or Vildagliptin [100 mg] or Linagliptin [5 mg]) and a full dose of Glimepiride (4 mg). Patients were stopped on these medications and shifted to a combination therapy with once-weekly dulaglutide ( $1.5 \mathrm{mg}$ or $0.75 \mathrm{mg}$ up-titrated to $1.5 \mathrm{mg}$ ), SGLT2i (canagliflozin [100 $\mathrm{mg}$ or $300 \mathrm{mg}$ ], empagliflozin [25 mg] or dapagliflozin [10 mg]) and metformin (500 mg or $1000 \mathrm{mg}$ ). Insulin glargine or degludec were subsequently required in 4 out of 15 patients to achieve a target FBS of 90-110 mg. The average dose of Basal Insulin was $8.35 \pm 0.45 \mathrm{IU}$. Patients with type 1 diabetes, history of pancreatitis, family history of medullary thyroid cancer, or multiple endocrine neoplasia type 2 were excluded from this study. All patients were on statins and antihypertensives (to achieve individualised optimal target SBP and DBP) in addition to antidiabetic medications. Mean SBP 122.87 $\pm 4.81 \mathrm{~mm} \mathrm{Hg}$ and mean DBP $84.27 \pm 4.95 \mathrm{~mm} \mathrm{Hg}$.

Data analysis was conducted for patients, who were able to complete three months of therapy.

\section{Endpoint assessment}

Changes in clinical parameters including, FBG, PPBG, $\mathrm{HbA}_{1 \mathrm{C}^{\prime}}$ body weight, BMI, VA, SBP and DBP were assessed at baseline and 3 months after treatment. AGEDIO B900 Pulse Wave Analysis System (Hibernia Medical). This instrument has a BP cuff that can be attached to the arms, a recording machine and a software which generates reports on the vascular age, based on the age, body weight, SBP and DBP and the arterial stiffness.

The report is generated in reference to the chronological age of the individual, i.e. same as or number of years more than or less than the chronological age.

Agedio is a screening service for arterial stiffness called VA measurement for the pharmacy store or other health care centers. The technology offers an (upperarm) cuff-based blood pressure and arterial stiffness measurement within one single procedure. Arterial stiffness is quantified with the aortic pulse wave velocity (PWV), measured in $\mathrm{m} / \mathrm{s}$. Agedio consists of a validated measurement device and the displaying device, an iPad. It allows for a measurement within a few minutes and provides two detailed reports one for the patient and one for the physician. The medical report in addition to arterial stiffness includes the hemodynamic figures such as cardiac output, total vascular resistance and central (aortic) blood pressure. 
Clinical indications for technology are as follows: Cardiovascular diseases such as coronary heart disease globally are the leading cause for morbidity and mortality. Risk assessment via blood pressure, LDL measurement or by using traditional risk scores such as Framingham and SCORE have shown to be insufficient for detecting the individual risk of a patient. The proportion of patients receiving inadequate treatment for cardiovascular diseases, such as high blood pressure, is alarmingly high. Existing measurement methods for blood pressure measurement do not provide enough medical information about the pathophysiological individual background of the patients.

Therefore, Agedio improves the current situation by following indications:

- leverage of patient awareness by using informative and catchy patient reports (pharmacy), supports the encouragement of patient therapy adherence;

- precise identification and classification of patients into low, medium and high risk patients (medical doctor);

- provision of hemodynamic figures for support in therapeutic decisions and thereby related clinical benefits, such as reduction of cardio-vascular events as strokes and myocardial infarctions (discovery).

The hemodynamic figures provide for a solid foundation of therapeutic decisions and benefits. They may provide the rationale for improved application for existing anti-hypertensive and other drugs. In comparison to regular therapy decision making, this advanced measure reduces emergency visits and re-hospitalization related to new cardiovascular and coronary events by $40 \%$.

The added value of risk prediction of PWV, above and beyond traditional risk factors, including SCORE and Framingham, has been quantified in a number of studies. The net classification value (NRI, net reclassification index) of PWV measurement allows for both, patient re-classification into higher or lower risk categories. The results have a direct influence on therapeutic decisions.

Agedio is a main point of action for stakeholders involved in public health, focusing on services within the pharmacy. Do you know your vascular age is a concept intended for a long-term partnership between pharmacists, medical professionals, health insurance companies and patients.

Adverse events following therapy administration were also recorded.

\section{Statistical methods}

All statistical analysis was performed using R 3.4.1 and MS-Excel software. Comparison between baseline
Table 1. Baseline demographics of the study group

\begin{tabular}{lc}
\hline Variable & $\begin{array}{c}\text { Baseline } \\
\text { Mean (SD) }\end{array}$ \\
\hline Age (years) & $49.47(9.29)$ \\
FBG [mg/dL] & $201.67(62.13)$ \\
PPBG [mg/dL] & $248.20(5.67)$ \\
$\mathrm{HbA}_{1 \mathrm{c}}(\%)$ & $8.61 \pm 1.41$ \\
Body weight $[\mathrm{kg}]$ & $98.60 \pm 14.05$ \\
BMI [kg/m²] & $32.27 \pm 4.67$ \\
VA (years) & $53.73 \pm 7.62$ \\
SBP [mm Hg] & $129.87 \pm 4.81$ \\
DBP [mm Hg] & $84.27 \pm 4.95$ \\
\hline
\end{tabular}

$\mathrm{BMI}$ - body mass index; $\mathrm{Cl}$ — confidence interval; $\mathrm{DBP}$ — diastolic blood pressure; FBG - fasting blood glucose; $\mathrm{HbA}_{1 \mathrm{c}}$ - glycosylated haemoglobin; LCL — lower confidence limit; PPBG — postprandial blood glucose; SBP - systolic blood pressure; SD - standard deviation; UCL - upper confidence limit; VA — vascular age

and follow-up value of various parameters was done using paired $t$ test. Continuous variables were presented as mean (standard deviation [SD]) with 95\% $\mathrm{Cl}$ as applicable and categorical data were presented as proportions. All $\mathrm{P}$ values were 2 -sided and were considered significant if $P<0.05$.

\section{Results}

Baseline demographics

A total of 15 (male: 9 and female: 6) patients received combination therapy with once-weekly dulaglutide, SGLT2i and metformin with or without insulin for 3 months. Baseline characteristics of the study population are presented in Table 1.

\section{Impact on clinical outcomes}

Improvement in clinical outcomes with dulaglutide as add-on therapy is presented in Table 2 .

\section{Glycaemic parameters}

A significant decline $(P<0.001)$ in FBG, PPBG and $\mathrm{HBA}_{1 \mathrm{c}}$ from baseline with a mean reduction (MR) of $66.67 \mathrm{mg} / \mathrm{dL}, 83.33 \mathrm{mg} / \mathrm{dL}$ and $1.78 \%$ respectively was demonstrated at 3-months follow-up.

\section{Non-glycaemic parameters}

\section{Body weight}

The therapy demonstrated a significant reduction $(P<0.001)$ in body weight (MR: $6.40 \mathrm{~kg})$ and corresponding BMI (MR: $2.67 \mathrm{~kg} / \mathrm{m}^{2}$ ) at 3-month follow-up.

\section{Vascular age}

The therapy was associated with a significant reduction ( $P<0.001$ ) in VA (MR: 3.93 years) at the follow-up. 
Table 2. Improvement in outcomes from baseline to follow-up in the overall population

\begin{tabular}{lccc}
\hline Variable & Mean improvement & Standard deviation & 95\%Cl (LCL, UCL) \\
\hline Glycaemic parameters & & & P value \\
FBG $[\mathrm{mg} / \mathrm{dL}]$ & 66.67 & 40.05 & $(46.40-86.93)$ \\
PPBG $[\mathrm{mg} / \mathrm{dL}]$ & 83.33 & 64.11 & $(50.89-115.78)$ \\
HbA $_{1 \mathrm{c}}(\%)$ & 1.78 & 1.08 & $(1.24-2.33)$ \\
Non-glycaemic parameters & & & $<.001$ \\
Body weight $[\mathrm{kg}]$ & 6.40 & 3.96 & $(4.40-8.40)$ \\
BMI $\left[\mathrm{kg} / \mathrm{m}^{2}\right]$ & 2.67 & 1.63 & $(1.84-3.49)$ \\
VA (years) & 3.93 & 2.46 & $(2.69-5.18)$ \\
SBP $[\mathrm{mm} \mathrm{Hg]}$ & 4.40 & 4.61 & $(2.07-6.73)$ \\
DBP $[\mathrm{mm} \mathrm{Hg]}$ & 2.80 & 3.84 & $<.001$ \\
\hline
\end{tabular}

$\mathrm{BMI}$ - body mass index; $\mathrm{Cl}$ - confidence interval; $\mathrm{DBP}$ - diastolic blood pressure; $\mathrm{FBG}$ — fasting blood glucose; $\mathrm{HbA}_{1 \mathrm{c}}$ - glycosylated haemoglobin; LCL — lower confidence limit; PPBG — postprandial blood glucose; SBP — systolic blood pressure; SD — standard deviation; UCL — upper confidence limit; VA - vascular age

\section{Blood pressure}

Follow-up at 3-months demonstrated a significant reduction $(P<0.05)$ in SBP (MR: $4.40 \mathrm{~mm} \mathrm{Hg}$ and DBP (MR: $2.80 \mathrm{~mm} \mathrm{Hg}$ ).

\section{Adverse events}

Self-reported gastrointestinal symptoms were observed in 5 (33.33\%) patients which included nausea and bloated abdomen. All adverse events subsided within 2 weeks of therapy initiation. Pantoprazole and domperidone combination were administered in 3 out of the 5 patients to mitigate the gastrointestinal side effects. No cases of severe hypoglycaemia were reported.

\section{Discussion}

Patients with T2DM often present metabolic and cardio-renal co-morbidities, adding complexity to diabetes care. With the advent of newer drugs such as GLP-1 RA and SGLT2i, the diabetes treatment is undergoing a paradigm shift towards improved diabetes care [7]. The current study evaluated the clinical effectiveness of combination therapy with once-weekly dulaglutide, SGLT2 $i$ and metformin with or without insulin in Indian adults with T2DM.

This study demonstrated significant reductions in glycaemic parameters such as FBG, PPBG, $\mathrm{HbA}_{1 c}$ for a follow-up period of 3 months. In addition, a significant reduction in body weight and BMI was observed. This is supported by one of the pivotal clinical trials for dulaglutide, AWARD-10 which compared the efficacy of combination therapy of dulaglutide and SGLT2i versus placebo and SGLT2 $i$ in patients with T2DM. Patients who received dulaglutide as an add-on to SGLT2i showed statistically superior improvements in glycaemic control compared with placebo and SGLT2i.
Similarly changes in weight, FBG and SBP was greater for the dulaglutide arms compared with placebo [9]. This finding is also in-line with another real-world study wherein the effectiveness of co-administration of GLP-1RA and SGLT2i was evaluated [7]. Notably, in a retrospective observational study by Curtis et al., which assessed the effect of combination of add-on therapy of GLP-1RA and SGLT2i on glycaemic control and weight in T2DM patients, reported significantly larger reduction in $\mathrm{HbA}_{1 c}$ with dual therapy (GLP-1RA and an SGLT2i) compared with a diabetic regimen with GLP-1RA alone. This effect was sustained along with the additional benefit of further weight loss in 58\% of the patients on dual add-on therapy compared to single GLP-1 therapy [10].

Clinically relevant improvement in daily and longterm glucose control with combined use of dulaglutide and SGLT2 inhibitors is important in the context of the differences in mechanisms of actions between GLP-1 receptor agonists and SGLT2 inhibitors - enhancement of insulin secretion and glucagon suppression with GLP-1 receptor agonists versus increased urinary glucose excretion and glucagon stimulation with SGLT2 inhibitors. Understanding the effect on glucagon was of special interest in this study because treatment with SGLT2 inhibitors has been shown to increase glucagon concentrations, potentially interfering with glucagon-lowering actions of GLP-1 receptor agonists. Importantly, both dulaglutide doses suppressed serum glucagon secretion, with dulaglutide $1.5 \mathrm{mg}$ achieving a significantly greater reduction from baseline versus placebo, consistent with the reductions in fasting glucagon concentration of similar magnitude reported in other studies of dulaglutide. The preserved effect of dulaglutide on glucagon when added to SGLT2 inhibi- 
tor treatment might be an important contributor to the overall glucose-lowering potency in this patient This preserved effect is not seen with dipeptidyl peptidase-4 (DPP-4) inhibitors or liraglutide when added to treatment with SGLT2 inhibitors since mean glucagon concentrations remained unchanged. This finding is potentially due to the lower potency of DPP-4 inhibitors and liraglutide compared with dulaglutide in the suppression of glucagon secretion [9]. The reduction in $\mathrm{HbA}_{1 \mathrm{c}}$ and bodyweight with dulaglutide $1.5 \mathrm{mg}$ in our study. One possible reason could be related to the simulteneous introduction of SGLT2 inhibitor therapy in patients on a background of medications which were weight neutral. Continuous decrease in $\mathrm{HbA}_{1 \mathrm{c}}$ and bodyweight suggest that the bodyweight-lowering action of simulteneously introduced SGLT2 inhibitors was ongoing during the treatment period.

A significant reduction in SBP and was noted at the 3-month follow-up. This is in contrast with a real-world study by Ghosal et al. which assessed the effectiveness of combination therapy with dulaglutide and SGLT2 $i$ in Indian patients with T2DM. However, the results are inline with clinical trial, AWARD-10 as elaborated above and other retrospective studies [7, 9].

Our study for the first time, reported the changes in vascular age as a result of combination therapy with dulaglutide and SGLT2i. Vascular age reflects the real atherosclerotic damage and is considered as a new variable in cardiovascular risk assessment [11, 12]. This study demonstrated significant improvement in vascular age at the follow-up compared with baseline. This is important as the assessment of cardiovascular risk is a cornerstone in effective prevention and management of cardiovascular disease often associated with T2DM.

Combination therapy was well tolerated; however, self-reported gastrointestinal symptoms subsided within 2 weeks of therapy initiation. There were no cases of severe hypoglycaemia reported in this study.

\section{Study limitations}

The most important limitation of this study is lack of a comparator arm. This leaves the possibility of risk of reporting bias that may affect the results. In addition, the study duration was relatively short (3 months). This study has a retrospective, single-centre observational design and small sample size. In an Indian setting, one of the barriers in accessing medications including GLP-1RA includes cost which limits the exposure of the drug to a narrow pool of patient population. Thus, these results might not be reflective of diverse patient population adding to selection bias. However, the importance of this study was not significantly affected by these limitations.

\section{Conclusion}

In a real-world setting, co-administration of dulaglutide with SGLT2i and other antidiabetic medications demonstrated significant improvements in glycaemic parameters and SBP. Interestingly, for the first time, the study reports improved vascular age with the therapy. The therapy was well tolerated with no serious adverse events reported. This study therefore provides a pilot and indeed generates a hypothesis supporting combination of GLP-1RA, SGLT-2i and other antidiabetic drugs, in the management of T2DM, which needs to be tested in a more systematic and appropriately powered study design. Further research is warranted in terms of healthcare resource practice, costs and long-term outcomes.

\section{Compliance with ethics}

All procedures were followed in accordance with the responsible committee on human experimentation and with the Helsinki Declaration of 1975 and subsequent revisions, and informed consent was received from the patient involved in this study.

\section{Acknowledgement}

The authors acknowledge Preethi Seshadri, Ph.D., and Kavya Shetty. MSc, Indegene Pvt. Ltd., for their writing assistance and editorial support.

\section{Conflict of interest}

The authors declare to have no conflict of interest.

\section{REFERENCES}

1. Busch RS, Kane MP. Combination SGLT2 inhibitor and GLP-1 receptor agonist therapy: a complementary approach to the treatment of type 2 diabetes. Postgrad Med. 2017; 129(7): 686-697, doi: 10.1080/00325481.2017.1342509, indexed in Pubmed: 28657399.

2. Kennedy M, Roberts A. Complex type 2 diabetes mellitus - management challenges and pitfalls. Aust Fam Physician. 2013; 42(4): 207-210, indexed in Pubmed: 23550246.

3. Zegarac JP. SGLT2 Inhibitor, GLP-1 receptor agonist combination treatment for type 2 diabetes: Expert insights. www endocrinologyadvisor com. https://www.endocrinologyadvisor.com/type2-diabetes-advisor/combination-sglt2-glucagon-like-peptide1-receptor-agonist-t2d/article/769123/. Accessed January 9, 2019.

4. van Baar MJB, van Ruiten CC, Muskiet MHA, et al. SGLT2 inhibitors in combination therapy: from mechanisms to clinical considerations in type 2 diabetes management. Diabetes Care. 2018; 41(8): 1543-1556, doi: $10.2337 / \mathrm{dc} 18-0588$, indexed in Pubmed: 30030256.

5. Gerstein HC, Colhoun HM, Dagenais GR, et al. REWIND Investigators. Dulaglutide and cardiovascular outcomes in type 2 diabetes (REWIND): a double-blind, randomised placebo-controlled trial. Lancet. 2019; 394(10193): 121-130, doi: 10.1016/S01406736(19)31149-3, indexed in Pubmed: 31189511.

6. Mody R, Grabner M, Yu M, et al. Real-world effectiveness, adherence and persistence among patients with type 2 diabetes mellitus initiating dulaglutide treatment. Curr Med Res Opin. 2018; 34(6): 
995-1003, doi: 10.1080/03007995.2017.1421146, indexed in Pubmed: 29271258.

7. Ghosal S, Sinha B. Liraglutide and Dulaglutide therapy in addition to SGLT-2 inhibitor and metformin treatment in Indian type 2 diabetics: a real world retrospective observational study. Clin Diabetes Endocrinol. 2018; 4: 11, doi: 10.1186/s40842-018-0061-8, indexed in Pubmed: 29760945.

8. Goncalves E, Bell DSH. Combination treatment of SGLT2 inhibitors and GLP-1 receptor agonists: symbiotic effects on metabolism and cardiorenal risk. Diabetes Ther. 2018; 9(3): 919-926, doi: 10.1007/s13300-018-0420-6, indexed in Pubmed: 29623594.

9. Ludvik B, Frías JP, Tinahones FJ, et al. Dulaglutide as add-on therapy to SGLT2 inhibitors in patients with inadequately controlled type 2 diabetes (AWARD-10): a 24-week, randomised, double- -blind, placebo-controlled trial. Lancet Diabetes Endocrinol. 2018; 6(5): 370-381, doi: 10.1016/S2213-8587(18)30023-8, indexed in Pubmed: 29483060.

10. Curtis L, Humayun M, Walker J, et al. Addition of SGLT2 inhibitor to GLP-1 agonist therapy in people with type 2 diabetes and suboptimal glycaemic control. Practical Diabetes. 2016; 33(4): 129-132, doi: 10.1002/pdi.2018.

11. Dakik HA. Vascular age for predicting cardiovascular risk: A novel clinical marker or just a mathematical permutation. J Nucl Cardiol. 2019; 26(4): 1356-1357, doi: 10.1007/s12350-018-1223-x, indexed in Pubmed: 29423908.

12. Łoboz-Rudnicka M, Jaroch J, Kruszyńska E, et al. Relationship between vascular age and classic cardiovascular risk factors and arterial stiffness. Cardiol J. 2013; 20(4): 394-401, doi: 10.5603/ CJ.2013.0098, indexed in Pubmed: 23913458. 